Article

\title{
Flexible Photonic Nanojet Formed by Cylindrical Graded-Index Lens
}

\author{
Cheng-Yang Liu $\mathbb{D}$ \\ Department of Biomedical Engineering, National Yang-Ming University, Taipei City 11221, Taiwan; \\ cyliu66@ym.edu.tw; Tel.: +886-2-28267020
}

Received: 18 March 2019; Accepted: 5 April 2019; Published: 7 April 2019

\begin{abstract}
Photonic nanojets formed in the vicinity of the cylindrical graded-index lens with different types of index grading are numerically investigated based on the finite-difference time-domain method. The cylindrical lens with $1600 \mathrm{~nm}$ diameter is assembled by eighty-seven hexagonally arranged close-contact nanofibers with $160 \mathrm{~nm}$ diameter. Simulation and analysis results show that it is possible to engineer and elongate the photonic nanojet. Using differently graded-index nanofibers as building elements to compose this lens, the latitudinal and longitudinal sizes of the produced photonic nanojet can be flexibly adjusted. At an incident wavelength of $532 \mathrm{~nm}$, the cylindrical lens with index grading $=2$ can generate a photonic nanojet with a waist about $173 \mathrm{~nm}$ ( 0.32 wavelength). This lens could potentially contribute to the development of a novel device for breaking the diffraction limit in the field of optical nano-scope and bio-photonics.
\end{abstract}

Keywords: cylindrical lens; photonic nanojet; graded-index

\section{Introduction}

Optical super-resolution has become significant for many applications including optical imaging [1,2], optical trapping and manipulation [3,4], nano-patterning and lithography [5,6], spectroscopy [7], and data storage [8]. Because the traditional objective lens has a diffraction-limited light spot, many investigations have been devoted to finding a practical way to obtain a small focusing spot beyond the diffraction limit [9]. One of the practical ways is the photonic nanojet (PNJ). The PNJ generated by an illuminated dielectric microcylinder is introduced and numerically demonstrated by Chen et al. in 2004 [10]. The mechanism of super-resolution imaging by dielectric microcylinders and microspheres have been increasingly attractive to researchers [11-15]. The PNJ is a high-intensity narrow focusing spot in the near-field of transparent microcylinder. When the diameter of transparent microcylinder is larger than the incident wavelength, the PNJ is generated due to the interferences between the scattering and illuminating fields. The main property of the PNJ is that it is a non-resonant phenomenon with low divergence and a small waist on the sub-wavelength scale. To generate a PNJ, it has been investigated that the refractive index contrast between the single microcylinder and its surrounding medium performs a critical role in the characters of PNJ [16]. This feature of microcylinder-based PNJ restricts the selection of transparent materials.

In order to optimize key parameters (focal length, waist, and intensity) of the PNJ, several studies indicate that the PNJ distributions depend on the geometric shape and refractive index of the microcylinder [17-24]. Moreover, the microcylinder or microsphere consisted of a concentric core-shell structure with different refractive indices for adjusting the propagation length and width of the PNJ [25-33]. The PNJ phenomenon can be changed significantly by applying shell materials with refractive index $>2$. The PNJ length formed by the core-shell microcylinder is increased to approximately 20 wavelengths. However, the price for this PNJ elongation is the waist widening and the intensity attenuation. The fabrication process of layered inhomogeneous core-shell microcylinder is 
very difficult and rather costly. Therefore, the new and simple procedure is an interesting research issue for PNJ shaping. The transparent medium in other geometries, such as micro-cuboids, micro-axicons, nanofibers, and optical fiber tips, are presented for the formation of PNJ [34-39]. These novel structures of transparent medium cause special features of PNJ-like intensity distributions and are highly probable to develop new applications.

In this paper, the combination of the metamaterial concept with the PNJ by plane wave illumination is proposed and numerically investigated. The graded-index nanofibers are used as building blocks to assemble the artificial cylindrical lens. By varying the graded-index type of the compositional nanofibers, the focusing properties of the lens are able to modulate according to our requirements. Using the finite-difference time-domain (FDTD) method, we simulated the optical field propagation of a plane wave passing through the cylindrical lens assembled by hexagonally arranged nanofibers in the air medium. The physical modeling is given in Section 2 for cylindrical graded-index lens. The effects of the graded-index types on the shape, focal length, full-width at half-maximum (FWHM), and intensity of PNJs are presented and discussed in Section 3. Finally, the conclusions of this investigation are summarized in Section 4.

\section{Physical Modeling}

Several numerical methods have theoretically studied optical intensity distribution in the vicinity of a transparent core-shell microcylinder or microsphere illuminated by a light source [26-29]. These studies suggest that the refractive index contrast between different shells plays a critical role in the formation of a PNJ. In order to verify the influence of the graded-index nanofibers, we performed FDTD calculations for modeling computational electromagnetics [40]. The schematic diagram of a PNJ generated by the cylindrical graded-index lens is shown in Figure 1. Geometrically, this cylindrical lens is constructed by multiple hexagonally arranged close-contact nanofibers which fully fill a cylindrical area with a particular diameter of $1600 \mathrm{~nm}$. The number and diameter of these nanofibers are 87 and $160 \mathrm{~nm}$. The proposed cylindrical lens is normally illuminated by a transverse electric plane wave propagating along $x$ direction with the electric field polarized along the $z$ direction. The length of this lens along the $z$ direction is defined as infinitely long for guaranteeing the accuracy and speed of FDTD calculation. The grid size of the FDTD mesh is chosen to be $10 \mathrm{~nm}$ after the convergence verification. The boundary conditions at the $x$ and $y$ directions added enough space to deliver the power flow distributions of optical beam in the background medium. The background medium is air with a refractive index of 1 .

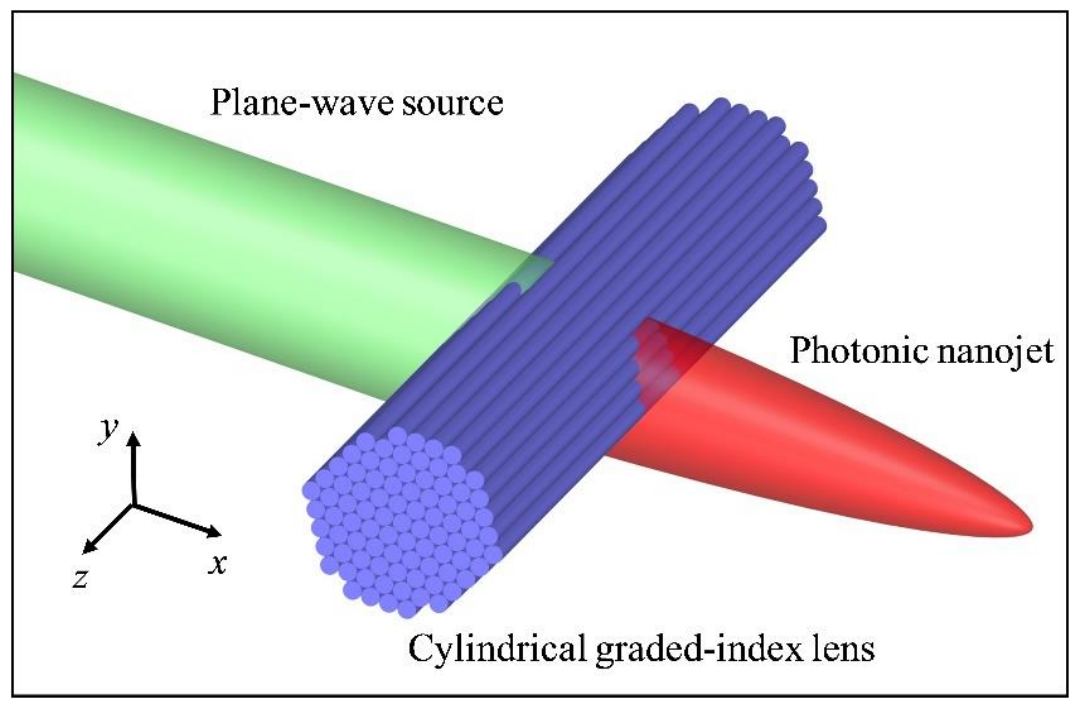

Figure 1. Schematic diagram of the cylindrical graded-index lens for photonic nanojet. 
Since the location and the intensity of PNJ depend on the refractive index contrast between each nanofiber layer, we consider a micrometer size cylindrical lens consisting of several concentric nanofibers with equal diameter. Figure $2 \mathrm{a}$ shows the graded-index model of the hexagonally arranged nanofibers. Every nanofiber layer with a number $s$ is a homogeneous material and is defined by the refractive index $n_{S}(s=0$ to $\mathrm{N})$. In order to specify the refractive index variation from layer to layer, the refractive index contrast of the cylindrical lens is expressed as $n_{s} / n_{0}=\left(n_{\mathrm{N}} / n_{0}\right)^{(s / \mathrm{N}) t}$ [26]. The index grading type parameter is $t$ and the dielectric central core is $s=0$. The $t>0$ indicates that the refractive index grading starts from the central nanofiber and terminates in the outermost nanofiber, which has the lowest value of the refractive index. Figure $2 b$ shows the different index grading types of refractive index $n_{s}$ in the graded-index lens. The $t$ value defines the variety of refractive index grading including linear $(t=1)$, concave $(t=0.2$ and $t=0.5)$, and convex $(t=1.5$ and $t=2)$ types. In the present lens, the refractive index grading is realized with $\mathrm{N}=5$ distinct concentric nanofibers. When the $t$ value is 1 , the refractive index grading is the linear layer-by-layer variation with the constant contrast. The maximum value of refractive index at the central nanofiber is 1.5 and it decreases in the radial direction to a minimum value of 1.05 at the outer nanofiber. This choice of index values is based on the practicability of modern micro-scale coating technology of objects with a thin film that has adjustable refractive indices [41,42]. The refractive indices in the range of 1.05 to 2.0 for material synthesis have been realized by controlling the porosity of silica glass. This manufacturing process is also possible to use for the graded-index photonic crystal structure and fiber.
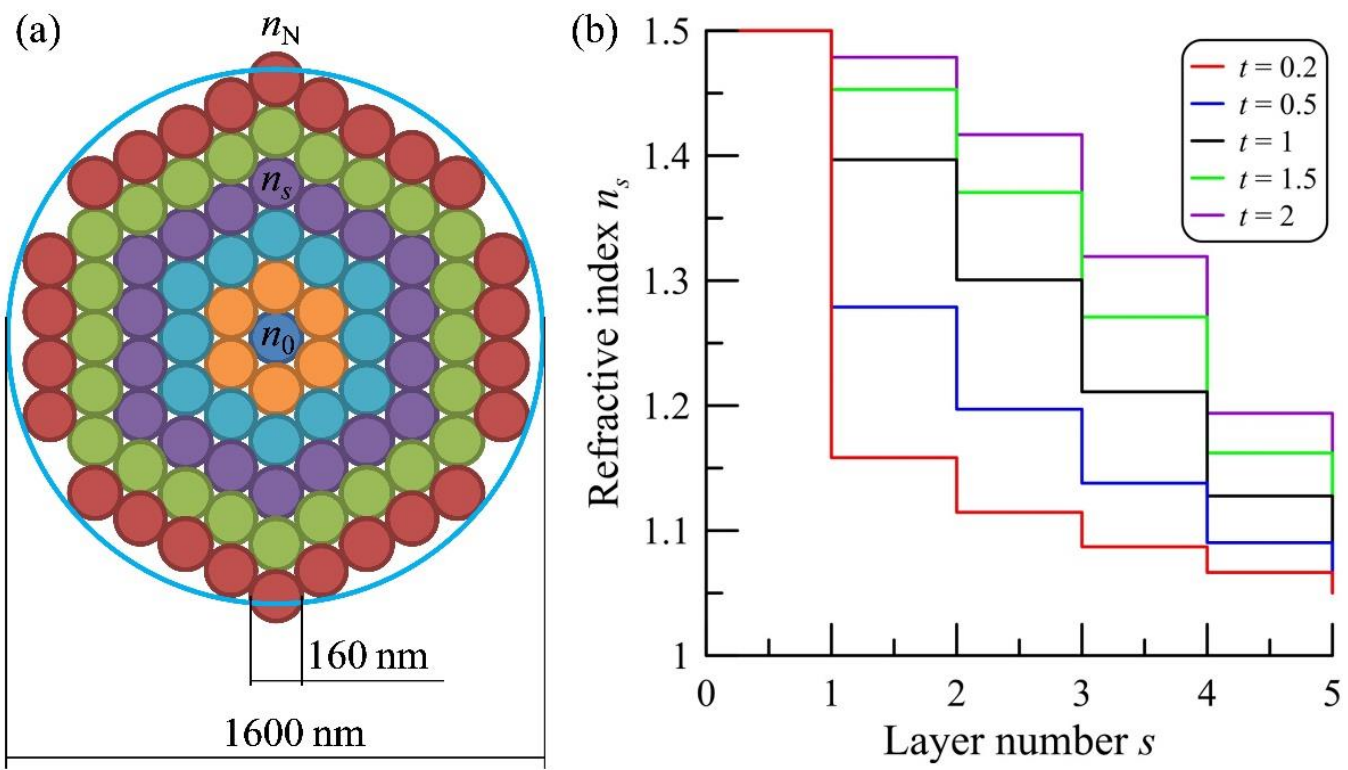

Figure 2. (a) Graded-index model of the hexagonally arranged nanofibers; (b) Different index grading types of refractive index $n_{s}$ in the graded-index lens.

\section{Results and Discussion}

The PNJ produced by a microcylinder has been found to present several important properties. First, the PNJ intensity is several hundred times higher than the incident light power. Second, the PNJ has a smaller waist than the classical diffraction limit. Using high-resolution FDTD calculation, we have simulated the intensity distributions of the cylindrical lens at different index grading types. The incident beam is linearly polarized with a wavelength of $532 \mathrm{~nm}$. Figure 3 shows the spatial intensity distributions of PNJs formed in the vicinity of cylindrical graded-index lens with homogeneous material $(n=1.5), t=0.2, t=0.5, t=1, t=1.5$, and $t=2$. Figure 3a represents the reference model at the same modeling conditions, which are the cylindrical lens with all homogeneous nanofibers. It demonstrated that an optical beam propagates from the top of the lens and the significant near-field focusing effect is observed at the bottom of the lens. Accordingly, an intensity peak of the electric field 
is known as the PNJ. The intensity peak in Figure 3a is 3.6 compared to the incident intensity of 1, and the FWHM of PNJ is $165 \mathrm{~nm}$ smaller than the incident wavelength of $532 \mathrm{~nm}$. The similar intensity distributions representing a cylindrical lens with nanofibers of five different index grading types are shown in Figure 3b-f. We could see that the PNJ gradually shifts from the outside to the inside of the lens when the index grading type parameter $t$ increases from 0.2 to 2 . The focusing effect plays a significant role in the propagation of the light wave in the lens and the PNJ is located at the interior of the nanofibers. Compared to the homogeneous model, PNJs created by different graded-index nanofibers assembled lens have stronger modulation of intensity peak and FWHM.
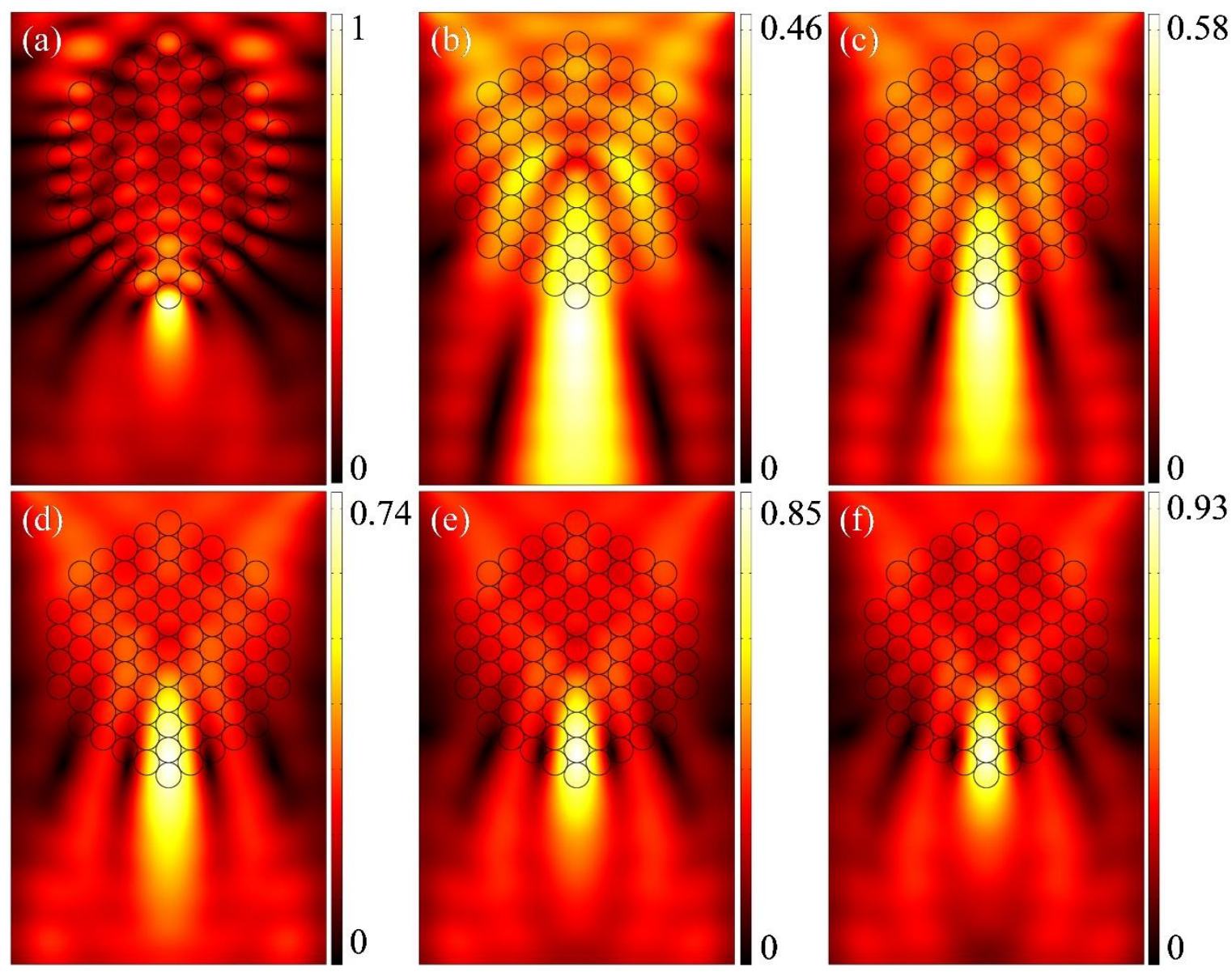

Figure 3. Spatial intensity distributions of photonic nanojets formed in the vicinity of cylindrical graded-index lens with (a) homogeneous material $(n=1.5),(\mathbf{b}) t=0.2,(\mathbf{c}) t=0.5,(\mathbf{d}) t=1$, (e) $t=1.5$, and (f) $t=2$.

Figure 4a shows the normalized intensity distributions of PNJ for cylindrical graded-index lens along the propagation axis ( $x$ axis). The longitudinal profile in Figure $4 \mathrm{a}$ is acquired as a two-dimensional cross-section of the intensity distribution by the straight line located at the center of the lens. The dashed line is the edge of the lens. According to Figure $4 \mathrm{a}$, the position of intensity peak for PNJ decreases from $1035 \mathrm{~nm}$ to $603 \mathrm{~nm}$ as the index grading type parameter increases. The transversal profiles at the highest intensity peak are plotted along the $y$ axis in Figure $4 \mathrm{~b}$. The FWHMs are $326 \mathrm{~nm}, 261 \mathrm{~nm}, 213 \mathrm{~nm}, 177 \mathrm{~nm}$, and $173 \mathrm{~nm}$ corresponding to $t=0.2,0.5,1,1.5$, and 2, respectively. The FWHM of the PNJ monotonically increases with the growth of the index grading type parameter as well. These indicate that the graded-index nanofibers are able to focus a light spot smaller than the Abbe diffraction limit. The smallest FWHM $(173 \mathrm{~nm})$ of the PNJ achieved by the model at $t=2$ is $35 \%$ smaller than the half of incident wavelength. Meanwhile, the highest intensity peak of the PNJ 
is delivered by the same graded-index nanofibers at $t=2$. These graded-index lens can be used in combination with a traditional objective lens for super-resolution imaging applications.
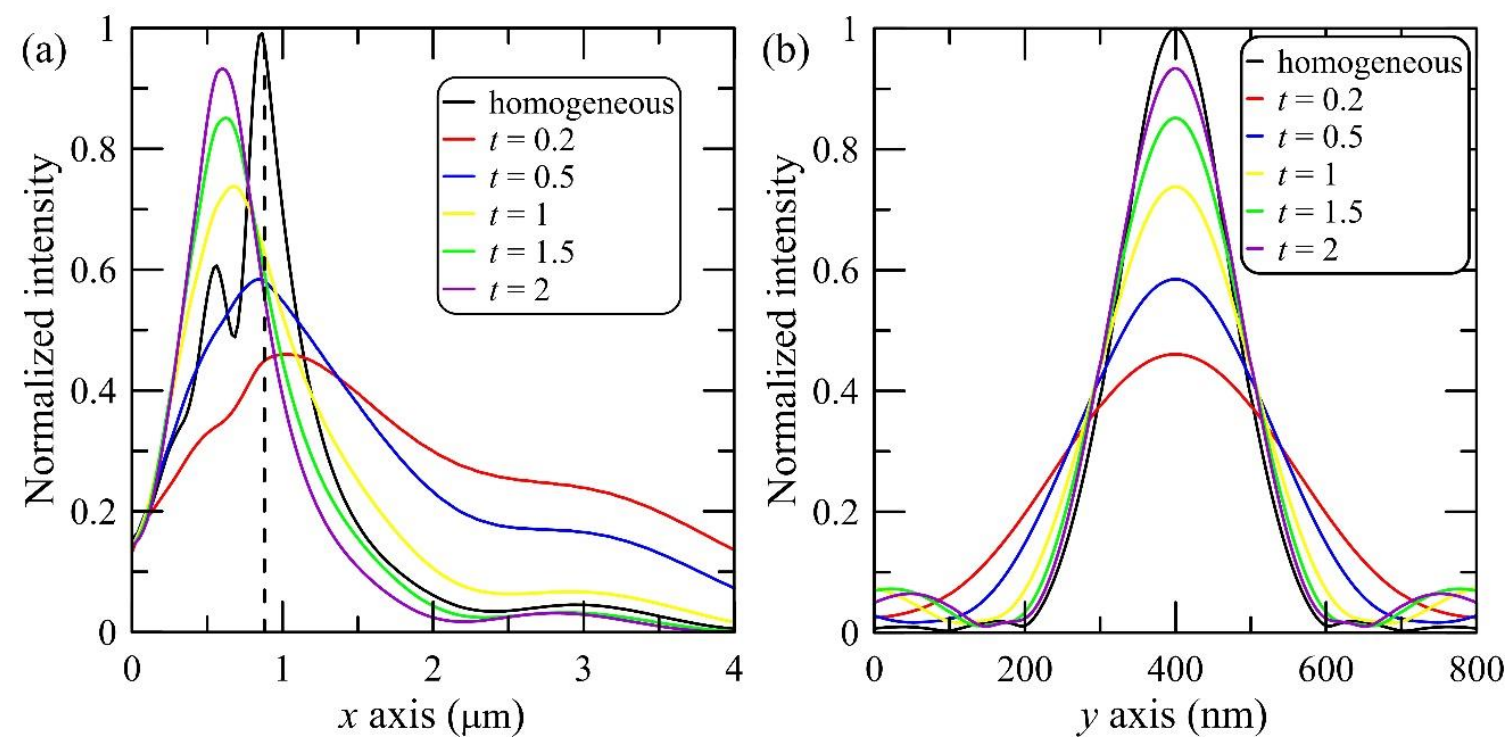

Figure 4. Normalized intensity distributions of photonic nanojet for cylindrical graded-index lens along (a) the propagation axis ( $x$ axis) and (b) the transversal axis ( $y$ axis). The dashed line is the edge of the lens.

If the PNJ is focused inside the lens, a magnified real image is formed as in the cases of Figure $3 \mathrm{~d}-\mathrm{f}$. It can be noted that the cylindrical graded-index lens produces a one-dimensional super-resolution image along the nanofiber axis. Therefore, we may obtain a complete two-dimensional super-resolution image in a large area by rotating the cylindrical lens in a circular mode. It is clear that the improved PNJ properties in the proposed graded-index lens originate from the introduced inhomogeneity of the refractive index. This graded-index lens is essentially a compact compound scattering media with altering refractive index along the propagation direction. Figure 5 shows the focal length and FWHM as a function of the index grading type parameter for cylindrical lens. Apparently, decreasing grading parameter $t$ results in elongated PNJ, accompanied by an expanded FWHM and decreased peak intensity. The evolution of the FWHM with the intensity peak with grading parameter t increasing from $326 \mathrm{~nm}$ to $173 \mathrm{~nm}$. Therefore, the key way to manipulate PNJ is to find an optimum graded-index configuration. If the grading parameter is $t>1$, the optical contrast of the serial nanofiber layers increases with their layer number. The central nanofiber plays the major function in the transformation of the PNJ inside the lens. When the grading parameter is $t>>1$, the graded-index lens according to its optical properties becomes similar to a homogeneous lens with high refractive index. 


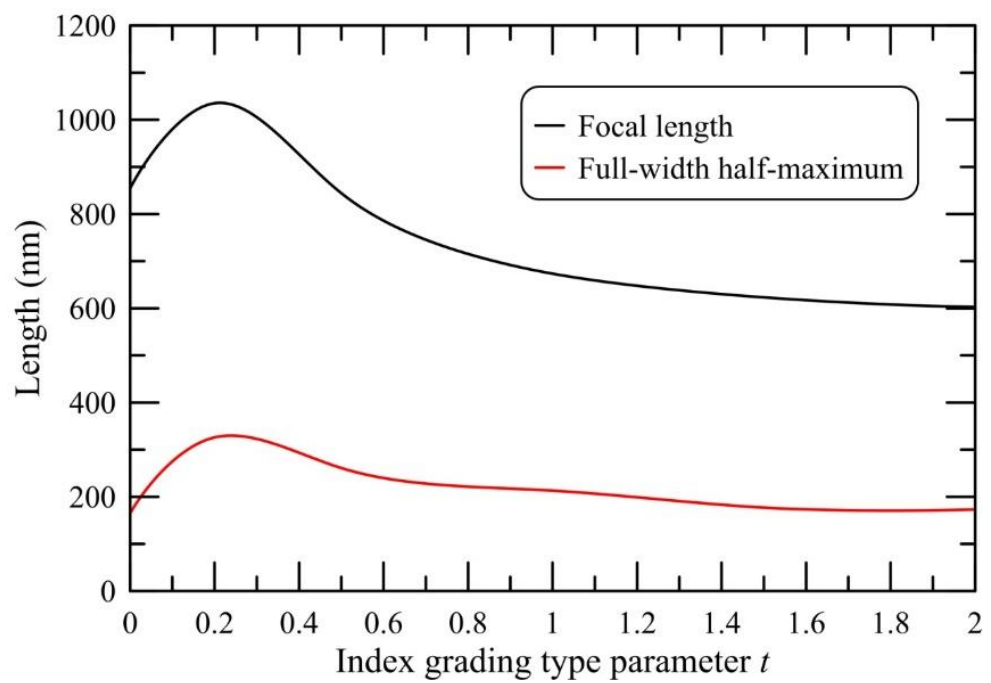

Figure 5. Focal length and full-width half-maximum as a function of the index grading type parameter for a cylindrical graded-index lens.

It can be seen from Figure 5 that the PNJ length decreases as $t$ value increases. The intensity peak is placed inside the nanofibers at the value of $t=2$. Combining basic properties of the PNJ, a modified quality criterion $\mathrm{Q}$ is expressed as $\mathrm{Q}=(\mathrm{L} \times \mathrm{I}) /$ FWHM [25]. The effective length, maximum peak intensity, and FWHM of the PNJ are L, I, and FWHM, respectively. The usability of a PNJ can be estimated by using this quality criterion in the solution of practical problems. When the $Q$ value is high, the peak intensity of nanojet is high, its FWHM is small, and the effective length is long. Figure 6 shows the quality criterion as a function of the index grading type parameter for cylindrical graded-index lens. At the value of $t=0.2$, the cylindrical graded-index lens optimally combines the high spatial localization with high intensity. The super-resolution and the relationship between the nanofibers and the light beam in the graded-index lens may have a physical connection with photonic crystal [43]. An individual nanofiber operates like a single nanolens. Due to the hexagonal arrangement, the grading refractive index is capable of guiding the intensity flow to the bottom nanofiber and generating a strong focus with a high-intensity peak. The nanofiber-assembled graded-index lens has some singular points which could be used to focus more power on the same phase. Therefore, this graded-index lens with the selected refractive index is suitable for nano-scale imaging.

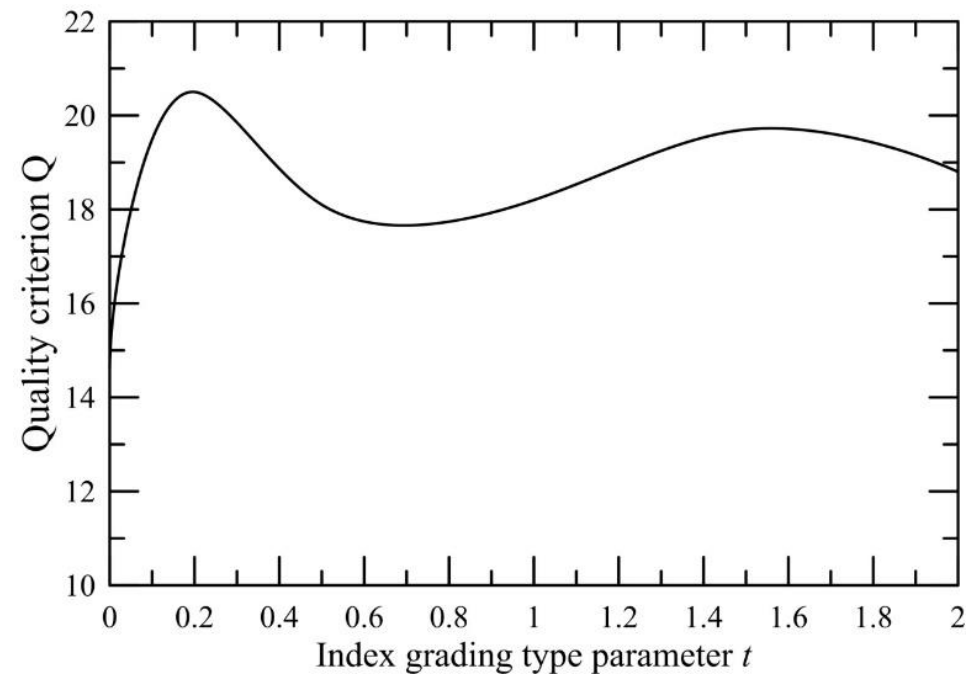

Figure 6. Quality criterion as a function of the index grading type parameter for the cylindrical graded-index lens. 
In order to compare the PNJ properties of our lens assembly to those of a single uniform-index microcylinder, we also performed FDTD calculation for a single microcylinder. The refractive index of a single microcylinder is 1.5. Spatial intensity distribution, normalized intensity distributions along the propagation axis and the transversal axis for PNJ formed by a single microcylinder with $1600 \mathrm{~nm}$ diameter are shown in Figure 7. In comparison with PNJ formed by cylindrical graded-index lens, it is noted that maximal intensity for the graded-index lens along the propagation axis is larger than peak intensity for uniform single microcylinder. Moreover, the FWHM for uniform single microcylinder is $277 \mathrm{~nm}$, but the FWHM for the graded-index lens at $t=0.2$ is $173 \mathrm{~nm}$. The focal length for uniform single microcylinder is $65 \mathrm{~nm}$ and the normalized peak intensity is 0.76 . The location of PNJ is close to the surface of the microcylinder.
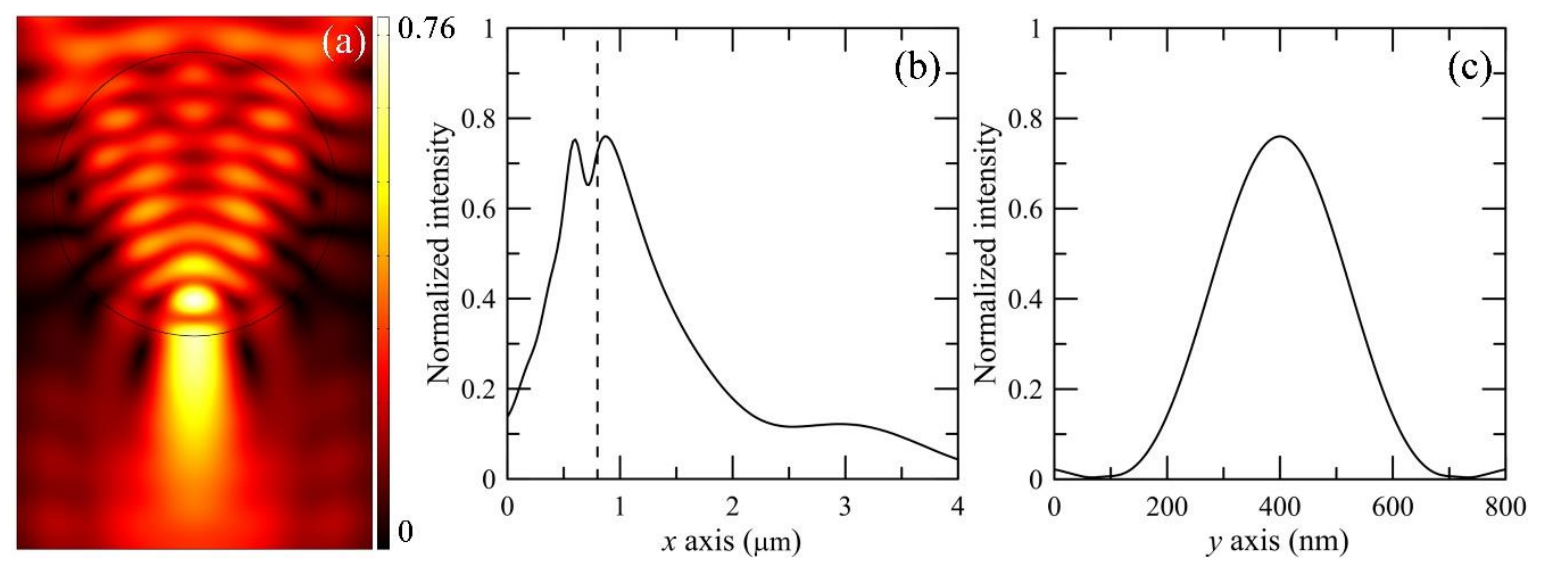

Figure 7. PNJ formed by a single microcylinder with $1600 \mathrm{~nm}$ diameter: (a) spatial intensity distribution, (b) normalized intensity distribution along the propagation axis, and (c) normalized intensity distribution along the transversal axis. The dashed line is the edge of the microcylinder.

\section{Conclusions}

In conclusion, the cylindrical graded-index lens assembled by hexagonally arranged transparent nanofibers is reported. The effective refractive index of the nanofibers can be changed by tuning the index grading type parameter. We are able to modulate the PNJ by varying the graded-index type of the compositional nanofibers. Using high-resolution FDTD calculation, we indicate that the PNJ is dynamically switched by the graded-index lens. Moreover, we present an optimization demonstration which pursues better focusing characters of the PNJ. The cylindrical graded-index lens can successfully achieve lateral resolution beyond the diffraction limit under the plane wave illumination of $532 \mathrm{~nm}$ wavelength. The hexagonal arrangement of the graded-index nanofibers leads to an alternating change of refractive index that effectively collects evanescent waves accompanied by near-field coupling of scattering light. Such a mechanism for PNJ manipulation may bring about new applications for optical imaging with super-resolution.

Funding: This research was funded by Ministry of Science and Technology of Taiwan, grant number MOST 107-2221-E-032-033.

Acknowledgments: The author would like to thank Igor V. Minin for his invaluable discussion.

Conflicts of Interest: The authors declare no conflict of interest.

\section{References}

1. Wang, Z.; Guo, W.; Li, L.; Lukyanchuk, B.; Khan, A.; Liu, Z.; Chen, Z.; Hong, M. Optical virtual imaging at $50 \mathrm{~nm}$ lateral resolution with a white-light nanoscope. Nat. Commun. 2011, 2, 218. [CrossRef] [PubMed]

2. Upputuri, P.; Pramanik, M. Microsphere-aided optical microscopy and its applications for super-resolution imaging. Opt. Commun. 2017, 404, 32-41. [CrossRef] 
3. Li, Y.; Xin, H.; Liu, X.; Zhang, Y.; Lei, H.; Li, B. Trapping and detection of nanoparticles and cells using a parallel photonic nanojet array. ACS Nano 2016, 10, 5800-5808. [CrossRef] [PubMed]

4. Shakhov, A.; Astaflev, A.; Nadtochenko, V. Microparticle manipulation using femtosecond photonic nanojet-assisted laser cavitation. Opt. Lett. 2018, 43, 1858-1861. [CrossRef]

5. Wu, W.; Katsnelson, A.; Memis, O.G.; Mohseni, H. A deep sub-wavelength process for the formation of highly uniform arrays of nanoholes and nanopillars. Nanotechnology 2007, 18, 485302. [CrossRef]

6. Jacassi, A.; Tantussi, F.; Dipalo, M.; Biagini, C.; Maccaferri, N.; Bozzola, A.; Angelis, F. Scanning probe photonic nanojet lithography. ACS Appl. Mater. Interfaces 2017, 9, 32386-32393. [CrossRef] [PubMed]

7. Li, X.; Chen, Z.; Taflove, A.; Backman, V. Optical analysis of nanoparticles via enhanced backscattering facilitated by 3-D photonic nanojets. Opt. Express. 2005, 13, 526-533. [CrossRef] [PubMed]

8. Kong, S.; Sahakian, A.V.; Heifetz, A.; Taflove, A.; Backman, A. Robust detection of deeply subwavelength pits in simulated optical data-storage disks using photonic jets. Appl. Phys. Lett. 2008, 92, 211102. [CrossRef]

9. Betzig, E.; Trautman, J.K. Near-field optics: Microscopy, spectroscopy, and surface modification beyond the diffraction limit. Science 1992, 257, 189-195. [CrossRef] [PubMed]

10. Chen, Z.; Taflove, A.; Backman, V. Photonic nanojet enhancement of backscattering of light by nanoparticles: a potential novel visible-light ultramicroscopy technique. Opt. Express 2004, 12, 1214-1220. [CrossRef]

11. Ferrand, P.; Wenger, J.; Devilez, A.; Pianta, M.; Stout, B.; Bonod, N.; Popov, E.; Rigneault, H. Direct imaging of photonic nanojets. Opt. Express 2008, 16, 6930-6940. [CrossRef]

12. Kim, M.; Scharf, T.; Mühlig, S.; Rockstuhl, C.; Herzig, H. Engineering photonic nanojets. Opt. Express 2011, 19, 10206-10220. [CrossRef] [PubMed]

13. Darafsheh, A.; Walsh, G.; Dal Negro, L.; Astratov, V. Optical super-resolution by high-index liquid-immersed microspheres. Appl. Phys. Lett. 2012, 101, 141128. [CrossRef]

14. Liu, C.; Wang, Y. Real-space observation of photonic nanojet in microspheres. Phys. E 2014, 61, 141-147. [CrossRef]

15. Darafsheh, A.; Bollinger, D. Systematic study of the characteristics of the photonic nanojets formed by dielectric microcylinders. Opt. Commun. 2017, 402, 270-275. [CrossRef]

16. Darafsheh, A. Influence of the background medium on imaging performance of microsphere-assisted super-resolution microscopy. Opt. Lett. 2017, 42, 735-738. [CrossRef] [PubMed]

17. Jalalia, T.; Ernib, D. Highly confined photonic nanojet from elliptical particles. J. Mod. Opt. 2014, 61, 1069-1076. [CrossRef]

18. Wu, M.; Huang, B.; Chen, R.; Yang, Y.; Wu, J.; Ji, R.; Chen, X.; Hong, M. Modulation of photonic nanojets generated by microspheres decorated with concentric rings. Opt. Express 2015, 23, 20096-20103. [CrossRef] [PubMed]

19. Wu, M.; Chen, R.; Soh, J.; Shen, Y.; Jiao, L.; Wu, J.; Chen, X.; Ji, R.; Hong, M. Super-focusing of center-covered engineered microsphere. Sci. Rep. 2016, 6, 31637. [CrossRef]

20. Liu, C.; Lin, F. Geometric effect on photonic nanojet generated by dielectric microcylinders with non-cylindrical cross-sections. Opt. Commun. 2016, 380, 287-296. [CrossRef]

21. Wu, M.; Chen, R.; Ling, J.; Chen, Z.; Chen, X.; Ji, R.; Hong, M. Creation of a longitudinally polarized photonic nanojet via an engineered microsphere. Opt. Lett. 2017, 42, 1444-1447. [CrossRef] [PubMed]

22. Yang, J.; Twardowski, P.; Gerard, P.; Duo, Y.; Fontaine, J.; Lecler, S. Ultra-narrow photonic nanojets through a glass cuboid embedded in a dielectric cylinder. Opt. Express 2018, 26, 3723-3731. [CrossRef]

23. Zhou, Y.; Gao, H.; Teng, J.; Luo, X.; Hong, M. Orbital angular momentum generation via a spiral phase microsphere. Opt. Lett. 2018, 43, 34-37. [CrossRef]

24. Chen, L.; Zhou, Y.; Wu, M.; Hong, M. Remote-mode microsphere nano-imaging: new boundaries for optical microscopes. Opto-Electron. Adv. 2018, 1, 170001. [CrossRef]

25. Kong, S.; Taflove, A.; Backman, V. Quasi one-dimensional light beam generated by a graded-index microsphere. Opt. Express 2009, 17, 3722-3731. [CrossRef]

26. Geints, Y.; Zemlyanov, A.; Panina, E. Photonic nanojet calculations in layered radially inhomogeneous micrometer-sized spherical particles. J. Opt. Soc. Am. B 2011, 28, 1825-1830. [CrossRef]

27. Liu, C. Superenhanced photonic nanojet by core-shell microcylinders. Phys. Lett. A 2012, 376, 1856-1860. [CrossRef]

28. Shen, Y.; Wang, L.; Shen, J. Ultralong photonic nanojet formed by a two-layer dielectric microsphere. Opt. Lett. 2014, 39, 4120-4123. [CrossRef] 
29. Wu, P.; Li, J.; Wei, K.; Yue, W. Tunable and ultra-elongated photonic nanojet generated by a liquid-immersed core-shell dielectric microsphere. Appl. Phys. Express 2015, 8, 112001. [CrossRef]

30. Minin, I.; Minin, O. Terahertz artificial dielectric cuboid lens on substrate for super-resolution images. Opt. Quant. Electron. 2017, 49, 326. [CrossRef]

31. Xing, H.; Zhou, W.; Wu, Y. Side-lobes-controlled photonic nanojet with a horizontal graded-index microcylinder. Opt. Lett. 2018, 43, 4292-4295. [CrossRef] [PubMed]

32. Liu, C.; Yen, T.; Minin, O.; Minin, I. Engineering photonic nanojet by a graded-index micro-cuboid. Phys. E 2018, 98, 105-110. [CrossRef]

33. Geints, Y.; Zemlyanov, A.; Minin, O.; Minin, I. Systematic study and comparison of photonic nanojets produced by dielectric microparticles in 2D- and 3D-spatial configurations. J. Opt. 2018, 20, 065606. [CrossRef]

34. Kotlyar, V.; Stafeev, S. Modeling the sharp focus of a radially polarized laser mode using a conical and a binary microaxicon. J. Opt. Soc. Am. B 2010, 27, 1991-1997. [CrossRef]

35. Geints, Y.; Zemlyanov, A.; Panina, E. Microaxicon generated photonic nanojets. J. Opt. Soc. Am. B 2015, 32, 1570-1574. [CrossRef]

36. Liu, C. Photonic jets produced by dielectric micro cuboids. Appl. Opt. 2015, 54, 8694-8699. [CrossRef]

37. Yue, L.; Yan, B.; Wang, Z. Photonic nanojet of cylindrical metalens assembled by hexagonally arranged nanofibers for breaking the diffraction limit. Opt. Lett. 2016, 41, 1336-1339. [CrossRef]

38. Yue, L.; Yan, B.; Monks, J.; Wang, Z.; Tung, N.; Lam, V.; Minin, O.; Minin, I. Production of photonic nanojets by using pupil-masked 3D dielectric cuboid. J. Phys. D: Appl. Phys. 2017, 50, 175102. [CrossRef]

39. Pierron, R.; Zelgowski, J.; Pfeiffer, P.; Fontaine, J.; Lecler, S. Photonic jet: key role of injection for etchings with a shaped optical fiber tip. Opt. Lett. 2017, 42, 2707-2709. [CrossRef] [PubMed]

40. Taflove, A.; Hagness, S. Computational Electrodynamics: The Finite Difference Time Domain Method; Artech House: Boston, MA, USA, 1998.

41. Poco, J.; Hrubesh, L. Method of producing optical quality glass having a selected refractive index. U.S. Patent 6,158,244, 2008.

42. Zhu, Q.; Fu, Y. Graded index photonic crystals: A review. Ann. Phys.-Berlin 2015, 527, 205-218. [CrossRef]

43. Ruskuc, A.; Koehler, P.; Weber, M.; Andres-Arroyo, A.; Frosz, M.; Russell, P.; Euser, T. Excitation of higher-order modes in optofluidic photonic crystal fiber. Opt. Express 2018, 26, 30245-30254. [CrossRef] [PubMed] 